DOI: http://dx.doi.org/10.3201/eid1801.111079

Suggested citation for this article: Hurt AC, Leang SK, Speers DJ, Barr IG, Maurer-Stroh S. Mutations I117V and I117M and oseltamivir sensitivity of pandemic (H1N1) 2009 viruses. Emerg Infect Dis [serial on the Internet]. 2012 Jan [date cited].

http://dx.doi.org/10.3201/eid1801.111079

\title{
Mutations I117V and I117M and Oseltamivir Sensitivity of Pandemic (H1N1) 2009 Viruses
}

Aeron C. Hurt, Sook Kwan Leang, David J. Speers, Ian G. Barr, and Sebastian Maurer-Stroh Author affiliations: World Health Organization Collaborating Centre for Reference and Research on Influenza, North Melbourne, Victoria, Australia (A.C. Hurt, S.K. Leang, I.G. Barr); PathWest Laboratory Medicine, Nedlands, Western Australia, Australia (D.J. Speers); Agency for Science, Technology and Research, Singapore (S. Maurer-Stroh); Ministry of Health, Singapore (S. Maurer-Stroh); and Nanyang Technological University, Singapore (S. Maurer-Stroh)

Analysis of mutations I117V and I117M in the neuraminidase of influenza A pandemic (H1N1) 2009 viruses showed that $1117 \mathrm{~V}$ confers a mild reduction in oseltamivir sensitivity and has a synergistic effect of further increasing resistance when combined with H275Y. Contrary to recent reports, the $1117 \mathrm{M}$ mutation does not alter oseltamivir sensitivity.

The neuraminidase inhibitors (NAIs) oseltamivir and zanamivir have been widely used in many countries to treat infection with influenza A pandemic (H1N1) 2009 virus. Since the start of the pandemic, $>27,000$ strains have been tested for oseltamivir resistance, of which 383 (1.4\%) have contained the $\mathrm{H} 275 \mathrm{Y}$ neuraminidase (NA) mutation, the same mutation that was found in oseltamivir-resistant prepandemic seasonal subtype $\mathrm{H} 1 \mathrm{~N} 1$ viruses that emerged in 2007-08. Apart from the H275Y mutation, a small number of other NAI resistance mutations have been detected in the NA of pandemic (H1N1) 2009 viruses, such as S247N (1), I223V (2), and I223R (3). Another NA mutation, I117M, has also recently been associated with oseltamivir resistance in pandemic (H1N1) 2009 viruses $(4,5)$. The I117M NA mutation was detected in a virus from South Korea that was isolated from a patient before oseltamivir treatment and then 
was detected, with the $\mathrm{H} 275 \mathrm{Y}$ mutation, in the same patient after oseltamivir treatment, although the dual mutations were not conclusively shown in the same virus (4,5). In both studies, neither the I117M variant nor the dual I117M + H275Y strain were specifically tested for oseltamivir resistance. Instead, the viruses were assumed to be resistant on the basis of previous studies that described a reduction in oseltamivir sensitivity in influenza A (H5N1) viruses because of an I117V amino acid substitution (6,7), rather than the I117M substitution detected in their studies.

Other studies have documented that different amino acid substitutions at key NA residues can cause considerably different effects on NAI susceptibility and that these mutations can have a variable effect in different NA backgrounds (8). Therefore, we tested the assumption that the I117M mutation confers oseltamivir resistance and investigate the role of the $1117 \mathrm{M}$ and the I117V mutations with and without the H275Y mutation in pandemic (H1N1) 2009 viruses.

\section{The Study}

By using site-directed mutagenesis and reverse genetics as described (9), we generated recombinant viruses with NA from the pandemic (H1N1) 2009 virus A/Auckland/1/2009 and the remaining genes from $\mathrm{A} / \mathrm{PR} / 8 / 34$. Recombinant viruses were constructed with no NA mutations, with single I117V or I117M NA mutations, and with dual I117V + H275Y or I117M + H275Y NA mutations.

NAI sensitivity analysis with a fluorescence-based NA inhibition assay (10) found that compared with a recombinant with no mutations, the I117V mutation conferred a 5-fold increase in the oseltamivir concentration required to inhibit $50 \%$ ( $\left.\mathrm{IC}_{50}\right)$ of the NA activity, a 2-fold increase in zanamivir $\mathrm{IC}_{50}$, and no change in peramivir $\mathrm{IC}_{50}$. In comparison, the $\mathrm{I} 17 \mathrm{M}$ mutation had no effect on sensitivity to any of the NAI drugs (Table 1).

The dual I117V + H275Y variant had oseltamivir and peramivir $\mathrm{IC}_{50}$ values that were $3 \times$ and $2 \times$ higher, respectively, than the $\mathrm{IC}_{50}$ of a virus with the $\mathrm{H} 275 \mathrm{Y}$ mutation alone. In contrast, the $\mathrm{IC}_{50}$ of the $\mathrm{I} 117 \mathrm{M}+\mathrm{H} 275 \mathrm{Y}$ variant was not substantially different from that of the $\mathrm{H} 275 \mathrm{Y}$ mutant for all of the NAIs, further demonstrating the lack of effect of the I117M mutation on NAI sensitivity. 
Analysis of 3,334 pandemic (H1N1) 2009 strains received at the World Health Organization (WHO) Collaborating Centre, Melbourne, Victoria, Australia, through the WHO Global Influenza Surveillance and Response System from April 2009 through June 2011, showed that 1 isolate had a I117V NA mutation, but no I117M variants were detected. The I117V variant, A/Perth/504/2010 (GenBank accession nos. HA: EPI279165 and NA: 279164; www.gisaid.com), was isolated from a 5-year-old boy, and had a 4-fold and 3-fold reduction in sensitivity to oseltamivir and zanamivir, respectively, similar to that of the RG-I117V strain (Table 1). Neither the patient nor his family members or siblings were undergoing any NAI treatment.

Apart from the A/Perth/504/2010 strain, no other pandemic (H1N1) 2009 strains with an I117V NA mutation were reported on GenBank or public sequence databases, demonstrating the high degree of conservation at this residue. However, 45 NA sequences from highly pathogenic influenza A (H5N1) strains in the public sequence databases contained the I117V mutation. The I117V mutation in highly pathogenic influenza A (H5N1) viruses has previously been reported to confer a 5- to 16-fold reduction in oseltamivir sensitivity and up to a 4-fold reduction in zanamivir sensitivity $(6,7)$.

Residue I117 is not in direct structural contact with oseltamivir, although it has neighboring residues that are known to affect drug susceptibility such as E119, R118, and V116. By using the predictive computational force field FoldX (11) in YASARA (12), we modeled the effects on structural stability of I117V, I117M, H275Y, I117V + H275Y, and I117M + H275Y mutations in the pandemic (H1N1) 2009 NA crystal structure (Protein Data Bank no. 3NSS; www.pdb.org) (13). The model estimated local destabilization effects for the known oseltamivirresistance mutation $\mathrm{H} 275 \mathrm{Y}$, which served as a control for the approach, whereas substantially smaller effects for I117V were observed, and almost no stability change was predicted for the I117M mutation (Table 2). The estimated local destabilization effects for the dual mutations $\mathrm{H} 275 \mathrm{Y}+\mathrm{I} 117 \mathrm{M}$ and H275Y + I117V were not substantially different from that predicted for the $\mathrm{H} 275 \mathrm{Y}$ mutation alone. When the NA inhibition assay $\mathrm{IC}_{50}$ data (Table 1) were compared with the estimated local destabilization effects of the mutants (Table 2), a good correlation was demonstrated between the 2 methods, although functional testing showed a larger difference between the $\mathrm{H} 275 \mathrm{Y}$ and the $\mathrm{H} 275 \mathrm{Y}+\mathrm{I} 117 \mathrm{~V}$ variants than that estimated in the computational model. 
The destabilization effect of I117V appears to be mainly caused by the increase in an internal cavity (Figure 1), which could increase flexibility of neighboring residues that form part of the drug-binding framework. The H275Y and I117V mutations are at opposite sides of the binding pocket (Figure 2) and, although they are not expected to affect each other's side-chain environment directly, the simultaneous changes on both sides of the drug show more effects on oseltamivir binding than the single mutations alone.

\section{Conclusions}

Although the I117V mutation was detected in 1 isolate from Australia, analysis of sequences from public databases shows that it is extremely rare in pandemic (H1N1) 2009 viruses to date. Although the I117V mutation causes a mild reduction in oseltamivir sensitivity, on the basis of pharmacokinetic data, we expect that a variant carrying this mutation would not be clinically resistant $(14,15)$. However, in combination with $\mathrm{H} 275 \mathrm{Y}$, the I117V mutation has a synergistic effect on oseltamivir resistance, raising the oseltamivir $\mathrm{IC}_{50}$ to $3 \times$ that caused by the $\mathrm{H} 275 \mathrm{Y}$ mutation alone and to a level that is likely to be clinically important. Previous studies have reported that the $\mathrm{I} 117 \mathrm{M}$ mutation may confer oseltamivir resistance $(4,5)$, although in this study we have demonstrated that this is not the case. These results therefore highlight the importance of assaying functional drug resistance when reporting novel mutations because resistance cannot be assumed on the basis of data from other amino acid substitutions at the same residue.

\section{Acknowledgments}

We thank the various laboratories that submitted specimens and isolates to the Melbourne WHO Collaborating Centre for Reference and Research on Influenza. We also thank Richard Webby for providing the reverse genetics plasmid pHW2000, Hoffmann-La Roche Ltd. (Basel, Switzerland) for providing oseltamivir carboxylate, GlaxoSmithKline (Melbourne, Victoria, Australia) for providing zanamivir, and BioCryst (Birmingham, AL, USA) for providing peramivir.

The Melbourne WHO Collaborating Centre for Reference and Research on Influenza is supported by the Australian Government Department of Health and Ageing.

Dr Hurt is a senior research scientist and head of the Antiviral Susceptibility Analysis Group within the WHO Collaborating Centre for Reference and Research on Influenza, Melbourne. His research interests include the role of key residues in NAI resistance and the effect of resistance mutations on viral replication and transmission. 


\section{References}

1. Hurt AC, Lee R, Leang S, Cui L, Deng Y, Phuah S, et al. Increased detection in Australia and Singapore of a novel influenza A(H1N1)2009 variant with reduced oseltamivir and zanamivir sensitivity due to a S247N neuraminidase mutation. Euro Surveill. 2011;16:pii:19884. PubMed

2. Santos L, Correia V, Giria M, Pedro S, Santos M, Silvestre M, et al. Genetic and antiviral drug susceptbility profiles of pandemic $\mathrm{A}(\mathrm{H} 1 \mathrm{~N} 1) \mathrm{v}$ influenza virus circulating in Portugal. In: Proceedings of Options for the Control of Influenza VII, Hong Kong, SAR, China, September 13, 2010. Atlanta (GA): Intregress; 2010. Abstract no. O-851.

3. van der Vries E, Stelma FF, Boucher CA. Emergence of a multidrug-resistant pandemic influenza A (H1N1) virus. N Engl J Med. 2010;363:1381-2. PubMed doi:10.1056/NEJMc1003749

4. Yi H, Lee JY, Hong EH, Kim MS, Kwon D, Choi JH, et al. Oseltamivir-resistant pandemic (H1N1) 2009 virus, South Korea. Emerg Infect Dis. 2010;16:1938-42. PubMed

5. Shin SY, Kang C, Gwack J, Kim JH, Kim HS, Kang YA, et al. Drug-resistant pandemic (H1N1) 2009, South Korea. Emerg Infect Dis. 2011;17:702-4. PubMed

6. Hurt AC, Selleck P, Komadina N, Shaw R, Brown L, Barr IG. Susceptibility of highly pathogenic $\mathrm{A}(\mathrm{H} 5 \mathrm{~N} 1)$ avian influenza viruses to the neuraminidase inhibitors and adamantanes. Antiviral Res. 2007;73:228-31. PubMed doi:10.1016/j.antiviral.2006.10.004

7. Ilyushina NA, Seiler JP, Rehg JE, Webster RG, Govorkova EA. Effect of neuraminidase inhibitorresistant mutations on pathogenicity of clade 2.2 A/Turkey/15/06 (H5N1) influenza virus in ferrets. PLoS Pathog. 2010;6:e1000933. PubMed doi:10.1371/journal.ppat.1000933

8. Hurt AC, Holien JK, Parker M, Barr IG. Oseltamivir resistance and the H274Y neuraminidase mutation in seasonal, pandemic and highly pathogenic influenza viruses. Drugs. 2009;69:252331. PubMed doi:10.2165/11531450-000000000-00000

9. Hurt AC, Holien JK, Barr IG. In vitro generation of neuraminidase inhibitor resistance in A(H5N1) influenza viruses. Antimicrob Agents Chemother. 2009;53:4433-40. PubMed doi:10.1128/AAC.00334-09

10. Hurt AC, Barr IG, Hartel G, Hampson AW. Susceptibility of human influenza viruses from Australasia and South East Asia to the neuraminidase inhibitors zanamivir and oseltamivir. Antiviral Res. 2004;62:37-45. PubMed doi:10.1016/j.antiviral.2003.11.008 
11. Van Durme J, Delgado J, Stricher F, Serrano L, Schymkowitz J, Rousseau F. A graphical interface for the FoldX forcefield. Bioinformatics. 2011;27:1711-2. PubMed doi:10.1093/bioinformatics/btr254

12. Krieger E, Koraimann G, Vriend G. Increasing the precision of comparative models with YASARA NOVA — a self-parameterizing force field. Proteins. 2002;47:393-402. PubMed doi:10.1002/prot.10104

13. Li Q, Qi J, Zhang W, Vavricka CJ, Shi Y, Wei J, et al. The 2009 pandemic H1N1 neuraminidase N1 lacks the 150-cavity in its active site. Nat Struct Mol Biol. 2010;17:1266-8. PubMed doi:10.1038/nsmb.1909

14. Peng AW, Milleri S, Stein DS. Direct measurement of the anti-influenza agent zanamivir in the respiratory tract following inhalation. Antimicrob Agents Chemother. 2000;44:1974-6. PubMed doi:10.1128/AAC.44.7.1974-1976.2000

15. He G, Massarella J, Ward P. Clinical pharmacokinetics of the prodrug oseltamivir and its active metabolite Ro 64-0802. Clin Pharmacokinet. 1999;37:471-84. PubMed doi:10.2165/00003088199937060-00003

Address for correspondence: Aeron C. Hurt, WHO Collaborating Centre for Reference and Research on Influenza, Victorian Infectious Diseases Reference Laboratory, 10 Wreckyn St, North Melbourne, 3051 Victoria, Australia; email: aeron.hurt@influenzacentre.org 
Table 1. NAI sensitivity of naturally occurring I117V mutant and I117V, I117M, I117V + H275Y, and I117M + H275Y variants from pandemic (H1N1) 2009 viruses compared with RG-WT virus*

\begin{tabular}{|c|c|c|c|c|c|c|c|}
\hline \multirow[b]{2}{*}{$\begin{array}{l}\text { Pandemic (H1N1) } 2009 \\
\text { viruses } \dagger\end{array}$} & \multirow[b]{2}{*}{ Mutation } & \multicolumn{2}{|c|}{ Zanamivir } & \multicolumn{2}{|c|}{ Oseltamivir carboxylate } & \multicolumn{2}{|c|}{ Peramivir } \\
\hline & & $\begin{array}{l}\text { Mean } \mathrm{IC}_{50} \pm \\
\mathrm{SD}, \mathrm{nmol} / \mathrm{L}\end{array}$ & $\begin{array}{c}\text {-fold } \\
\text { differencef }\end{array}$ & $\begin{array}{l}\text { Mean } \mathrm{IC}_{50} \pm \\
\mathrm{SD}, \mathrm{nmol} / \mathrm{L}\end{array}$ & $\begin{array}{c}\text {-fold } \\
\text { difference }\end{array}$ & $\begin{array}{l}\text { Mean } \mathrm{IC}_{50} \pm \\
\mathrm{SD}, \mathrm{nmol} / \mathrm{L}\end{array}$ & $\begin{array}{c}\text {-fold } \\
\text { difference }\end{array}$ \\
\hline $\begin{array}{l}\text { Mean of NAl-sensitive } \\
\text { viruses }\end{array}$ & - & $0.28 \pm 0.15$ & - & $0.45 \pm 0.35$ & - & $0.20 \pm 0.10 \S$ & - \\
\hline A/Perth/504/2010 & I117V & $0.96 \pm 0.28$ & 3 & $1.63 \pm 0.70$ & 4 & $0.09 \pm 0.01$ & 1 \\
\hline RG-WT & - & $0.24 \pm 0.05$ & - & $0.30 \pm 0.20$ & - & $0.09 \pm 0.01$ & - \\
\hline RG-I117V & I117V & $0.54 \pm 0.12$ & 2 & $1.42 \pm 0.52$ & 5 & $0.08 \pm 0.02$ & 1 \\
\hline RG-I117M & I117M & $0.32 \pm 0.05$ & 1 & $0.31 \pm 0.06$ & 1 & $0.11 \pm 0.01$ & 1 \\
\hline$R G-I 117 V+H 275 Y$ & $\begin{array}{l}\mathrm{I} 117 \mathrm{~V}+ \\
\mathrm{H} 275 \mathrm{Y}\end{array}$ & $0.44 \pm 0.05$ & 2 & $568.84 \pm 54.89$ & 1,896 & $47.08 \pm 32.57$ & 523 \\
\hline RG-I117M + H275Y & $\begin{array}{l}\mathrm{I} 117 \mathrm{M}+ \\
\mathrm{H} 275 \mathrm{Y}\end{array}$ & $0.29 \pm 0.04$ & 1 & $163.72 \pm 17.76$ & 546 & $16.12 \pm 0.84$ & 179 \\
\hline RG-275Y & $\mathrm{H} 275 \mathrm{Y}$ & $0.26 \pm 0.03$ & 1 & $195.02 \pm 21.05$ & 650 & $19.72 \pm 1.42$ & 219 \\
\hline
\end{tabular}

${ }^{*} \mathrm{NAI}$, neuraminidase inhibitor; $\mathrm{IC}_{50}, 50 \%$ inhibitory concentration; -, not applicable.

$+n=3,334$, obtained through World Health Organization Global Influenza Surveillance and Response System, April $2009-J u n e 2011$.

‡-fold differences compared with $I_{50}$ value of RG-WT. Calculated for A/Perth/504/2010 on the basis of comparison to the mean IC 50 of circulating pandemic (H1N1) 2009 viruses, whereas the $I_{50}$ values of the modified RG viruses were compared to the IC 50 of the RG-WT virus.

$\S$ Mean and SD of peramivir $I_{50}$ values were based on analysis of 273 isolates. RG strains were derived by using site-directed mutagenesis and reverse genetics. Mean $I_{50} \pm$ SD values of the A/Perth/504/2010 virus and the RG strains were calculated on the basis of values derived from 3 independent assays.

Table 2. Predicted local structure destabilization for the different NA mutations from pandemic (H1N1) 2009 viruses, obtained during April 2009-June 2011*

\begin{tabular}{lc}
\hline $\mathrm{NA}$ mutation & Mean \pm SD level of destabilization, $\mathrm{kcal} / \mathrm{mol} \dagger$ \\
\hline $\mathrm{H} 275 \mathrm{Y}$ & $4.7 \pm 0.4$ \\
$\mathrm{H} 275 \mathrm{Y}+\mathrm{I117V}$ & $5.2 \pm 0.4$ \\
$\mathrm{H} 275 \mathrm{Y}+\mathrm{I117M}$ & $5.0 \pm 0.3$ \\
$\mathrm{I} 117 \mathrm{~V}$ & $0.5 \pm 0.02$ \\
$\mathrm{I} 117 \mathrm{M}$ & $0.1 \pm 0.1$ \\
\hline${ }^{*} \mathrm{NA}$, neuraminidase. &
\end{tabular}

†Calculated with FoldX (11) on the basis of 5 repetitions. 


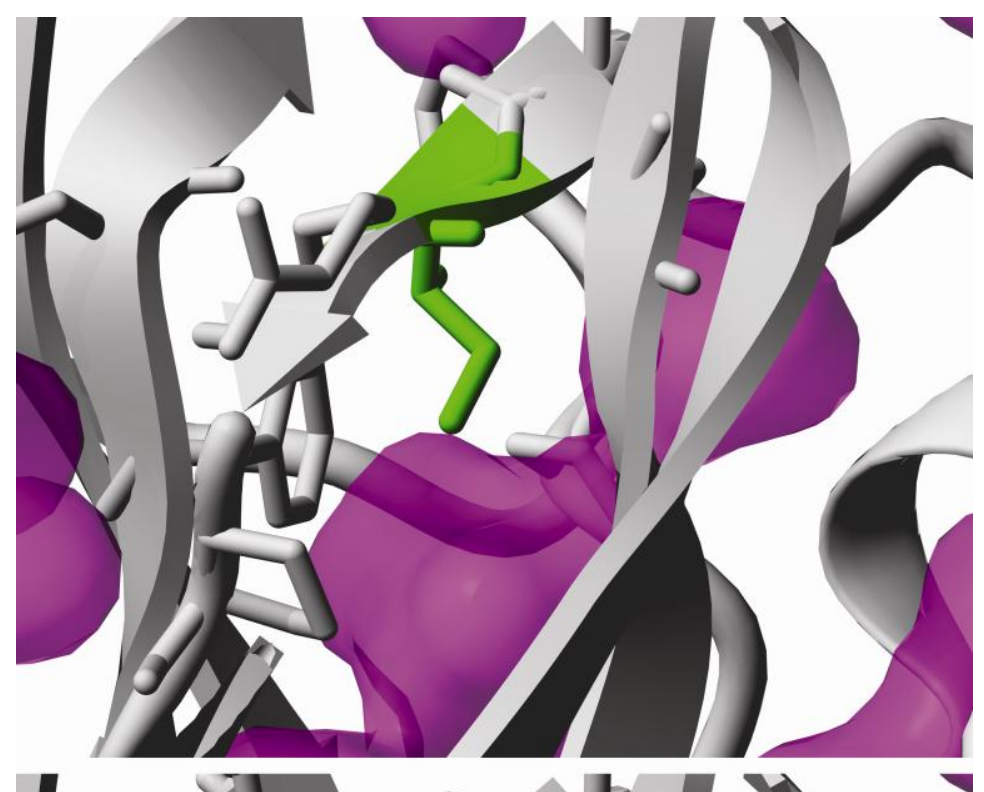

Figure 1. Structural details of neuraminidase mutations from pandemic (H1N1) 2009 viruses. Top, wildtype mutation 1117 (green); middle, mutation I117V (red); bottom, mutation I117M (blue). All were modeled with FoldX (11) in YASARA (12) in the context of the pandemic (H1N1) 2009 virus neuraminidase crystal structure (Protein Data Bank: 3nss). Side chains of residues $\leq 3$ Ångström of residue 117 are shown as sticks. Cavities within the structure (1.4 Å radius water probe) are shown in magenta.

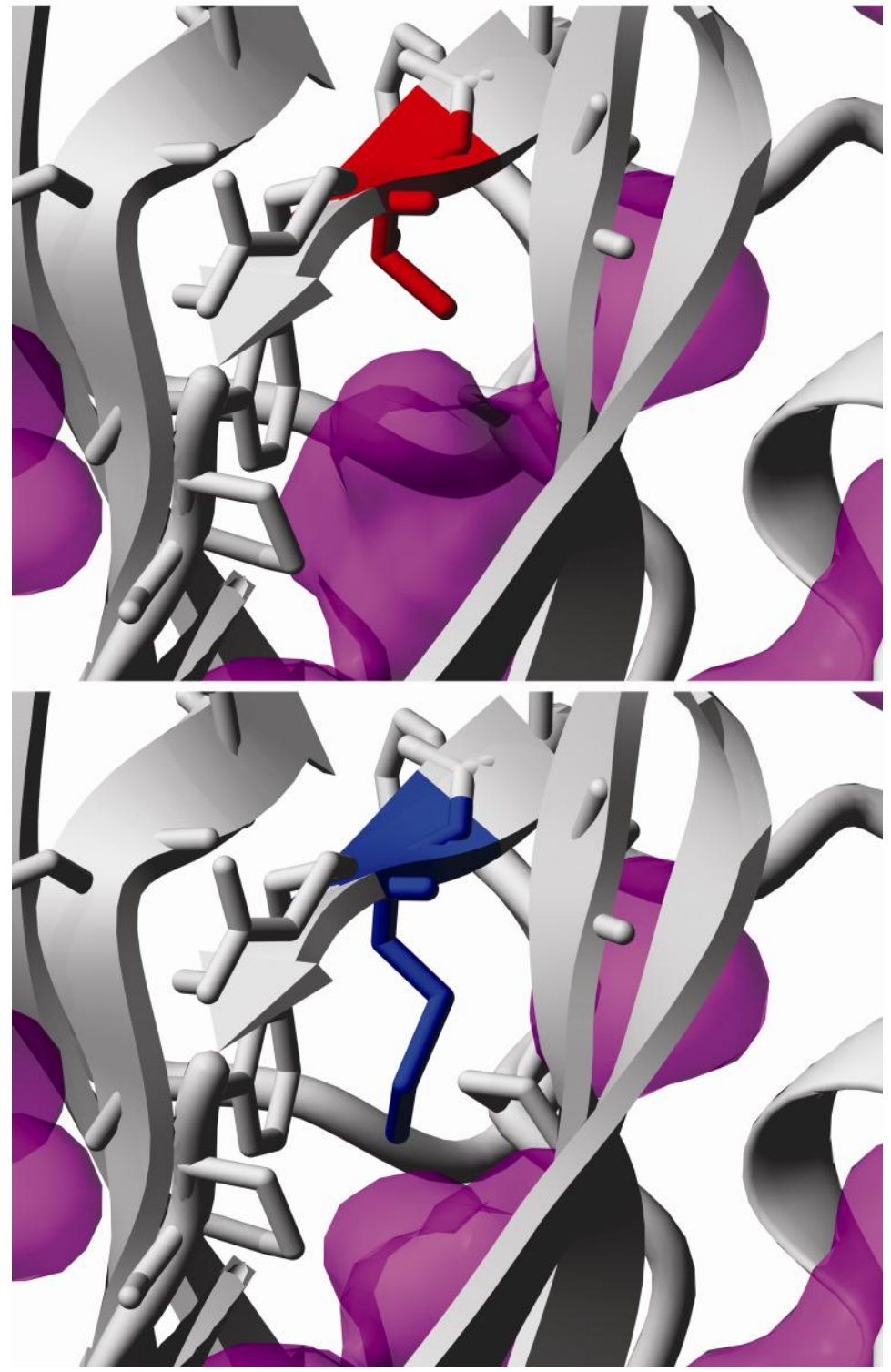

Page 8 of 9 


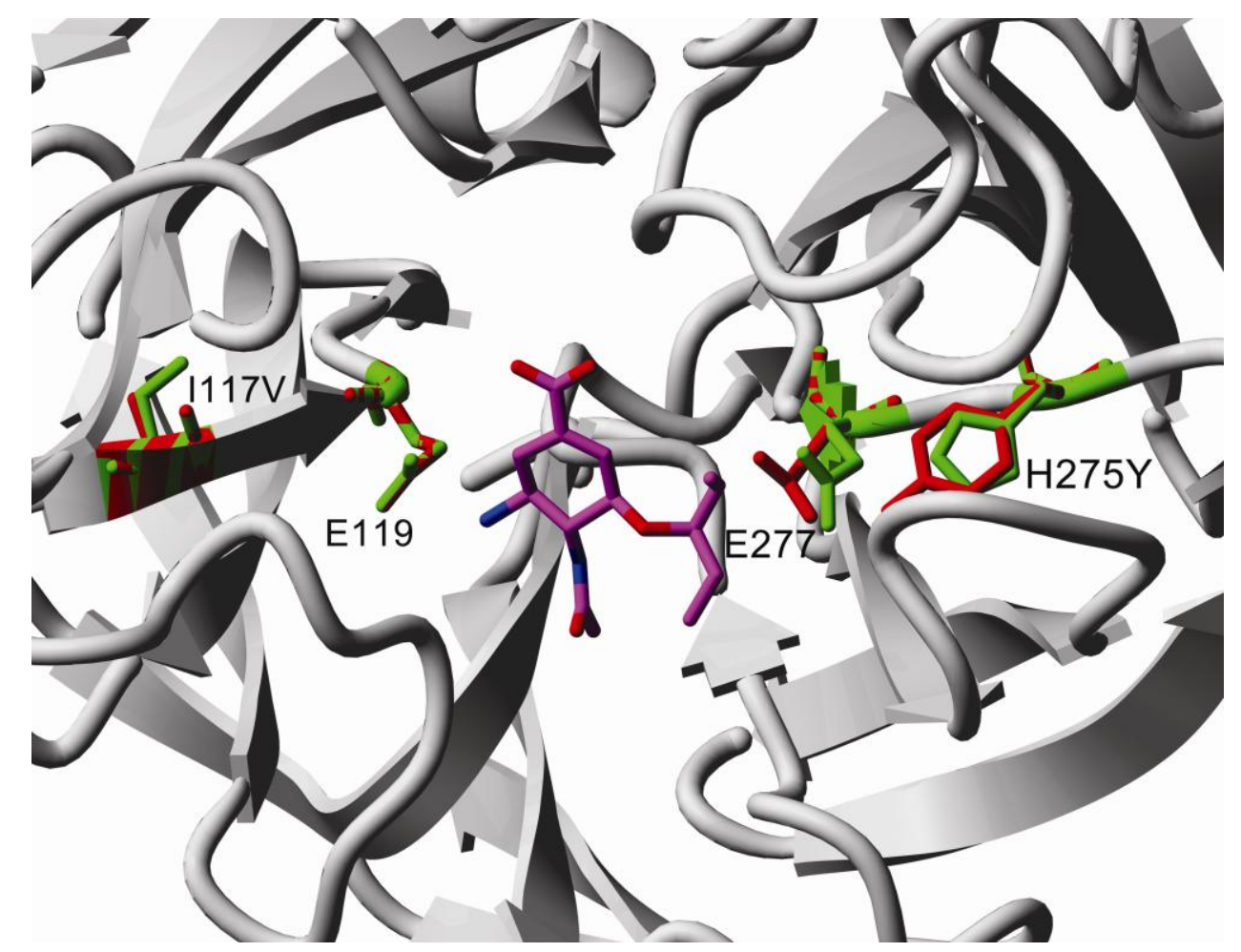

Figure 2. Comparison of wildtype I117 mutation from pandemic (H1N1) 2009 viruses (green residues; Protein Data Bank: 3nss) with FoldX/YASARA model of I117V + H275Y double mutant (red residues) $(11,12)$. Residue numbering is according to pandemic (H1N1) 2009 neuraminadase. Oseltamivir is added as reference (Protein Data Bank: $3 \mathrm{clO}$ ) and shown in magenta. 